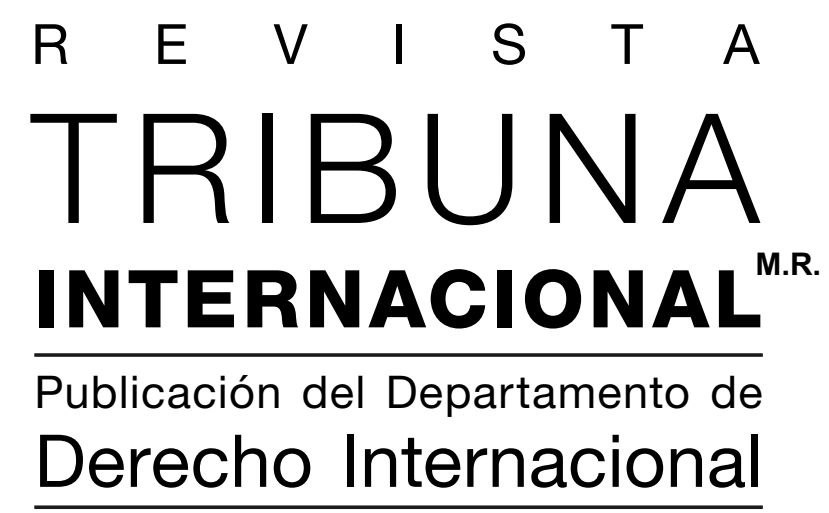

Volumen $4 / N^{\circ} 7 / 2015$

DERELTAD DE

UNIVERSIDAD DE CHILE 


\section{Rector de la Universidad de Chile}

Ennio Vivaldi Véjar

Av. Alameda Libertador Bernardo

O’Higgins 1058, Santiago

Representante legal

Davor Harasic Yaksic

Decano de la Facultad de Derecho

Universidad de Chile

Director del Departamento de

Derecho Internacional

Edmundo Vargas Carreño

Director de la Revista Tribuna

Internacional

Mario Ramírez Necochea

Editor General de la Revista Tribuna

Internacional

Luis Valentín Ferrada Walker

\section{Comité Editorial}

Íñigo Álvarez Gálvez (Universidad de Chile, Chile)

Gonzalo Aguilar (Universidad de Talca, Chile)

José Carlos Fernández Rosas

(Universidad Complutense de Madrid,

España)

Claudio Grossman (American

University, EE.UU)

Mattias Kumm (New York University,

EE.UU)

Hugo Llanos (Universidad Central, Chile)

Cecilia Medina (Universidad Diego

Portales, Chile)

Elina Mereminskaya (Universidad de Chile, Chile)

Mónica Pinto (Universidad de Buenos Aires, Argentina)

\section{Revista Tribuna Internacional M.R.}

Publicación del Departamento de Derecho Internacional de la Facultad de Derecho de la Universidad de Chile. $\mathrm{Su}$ objetivo es fomentar la reflexión, el debate, el análisis y la comunicación sobre el derecho internacional en forma pluralista y con rigor científico. Se publica cada semestre en los meses de junio y diciembre mediante convocatoria abierta a la publicación de artículos y monografías inéditos, comentarios de jurisprudencia, recensiones y comentarios de libros, en los campos de derecho internacional público y privado, derecho internacional de los derechos humanos y relaciones internacionales, tanto en castellano como en inglés.

Volumen 4/ No 7 / 2015

www.tribunainternacional.uchile.cl

ISSN 0719-210X (versión impresa)

ISSN 0719-482X (versión en línea)

Departamento de Derecho Internacional Facultad de Derecho

Universidad de Chile

Av. Santa María 076, $4^{\circ}$ piso

Providencia, Santiago de Chile

\section{Diseńo y producción:}

Gráfica LOM

www.lom.cl

Impreso en Chile/ Printed in Chile

Se autoriza la reproducción total o parcial del contenido de la publicación, siempre que se reconozca y cite el/ la/ los/ las autor/a/es/as y la publicación, no se realicen modificaciones a la obra y no se la utilice para fines comerciales. 
Revista Tribuna Internacional

Volumen $4 \bullet \mathrm{N}^{0} 7 \bullet 2015 \bullet$ pp. 69-86

ISSN 0719-210X (versión impresa)/ISSN 0719-482X (versión en línea)

\title{
La Responsabilidad Democrática en las Américas: Un mandato compartido ${ }^{1}$
}

\author{
Democratic Responsibility in the Americas: A shared \\ mandate
}

\section{Moisés A. Montiel M.}

mmontielm88@gmail.com

Abogado egresado de la Universidad Central de Venezuela. Maestro de Leyes en Derecho Internacional del Fletcher School of Law and Diplomacy. Tufts University, Massachusetts, Estados Unidos.

Resumen: La Carta Democrática Interamericana contiene el compromiso individual de los países de las Américas con la construcción de democracias sólidas y estables. El presente estudio busca dilucidar si de dicho instrumento puede extraerse la existencia de una obligación jurídica común a los Estados Miembros de la Organización de Estados Americanos en construir colectiva y cooperativamente tal cometido. Considerando la entidad del Derecho a la Democracia y trasladando la doctrina de la Responsabilidad de Proteger al contexto de la Democracia Prescriptiva Americana, teorizaremos sobre su aplicabilidad y su situación a la luz del Principio de No Intervención cuando un Estado americano no quiere o no puede cumplir con su obligación democrática.

Palabras claves: democracia, Responsabilidad de Proteger, Organización de Estados Americanos y Derecho Interamericano.

Abstract: The Democratic Inter-American Charter stipulates the individual commitment of American States in the construction of solid and stable democracies. This study seeks to elucidate if a legal obligation to the collective and cooperative construction of that goal, common to all Member States of the Organization of American States, can be extracted from said instrument. Considering the entity of the Right to Democracy and applying the doctrine of the Responsibility to Protect in the context of the Prescriptive American Democracy we will inquire on its applicability and its situation in light of the Principle of Non Intervention when an American State is unable or unwilling to fulfill its democratic obligation.

Keywords: Democracy, Responsibility to Protect, Organization of American States, Inter-American Law.

$1 \quad$ Artículo enviado el 13.02.2015 y aceptado el 03.06.2015. 


\section{Introducción}

La Carta Democrática Interamericana (en adelante CDI) es el instrumento sancionado por la Organización de Estados Americanos (en adelante OEA) en el cual consta el compromiso de los Estados americanos para la construcción y sostenimiento de democracias $^{2}$. En dicho instrumento se consagra el denominado Derecho a la Democracia, a la vez que reconoce la obligación de sus gobiernos en su promoción y defensa. La misma, según refiere Aguiar, es "la obra de una larga maduración sobre los predicados de la misma democracia, según el entendimiento que de ella tienen la doctrina política y judicial regional"3. En función de tal maduración, los gobiernos del continente americano convinieron en aceptar la CDI como un "instrumento jurídicamente vinculante por ser interpretación auténtica bien de la Carta de la Organización de Estados Americanos

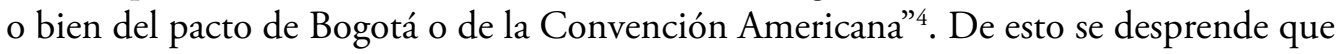
la CDI es fuente auténtica y de obligatorio cumplimiento del Derecho Interamericano en tanto interpretación autorizada de un tratado internacional válidamente celebrado por los Estados que componen la OEA. En este sentido, la buena fe ha de prevalecer en la ejecución de cualquier tratado en vigor, visto que atiende a la satisfacción de la necesidad de seguridad jurídica en la sociedad internacional ${ }^{5}$.

Partiendo de la premisa del carácter vinculante - por asociación a la Carta de la OEApara los Estados americanos del denominado Derecho a la Democracia, convendrá analizar cuál es su entidad. En este sentido, el objeto de estudio del presente trabajo es analizar sí existe una obligación colectiva de los Estados americanos en la construcción de democracias que sigan los parámetros delineados por la CDI.

Tradicionalmente, la práctica gubernamental americana en materia de democracia ha parecido entender que se trata de un asunto esencialmente doméstico. A tal efecto se ha escudado férreamente en el Principio de No Intervención. A despecho de esto, si se analizan cuidadosamente distintos instrumentos propios del Derecho Interamericano,

2 Aprobada mediante resolución de la Asamblea General de la OEA, en la primera sesión plenaria de su $28^{\circ}$ Período de Sesiones Extraordinarias, en Lima, Perú, el 11 de septiembre de 2001.

3 Aguiar, Asdrúbal. La Democracia en la jurisprudencia de la Corte Interamericana de Derechos Humanos 1987-2012. Buenos Aires/Caracas, Observatorio Iberoamericano de la Democracia, 2012. [en línea] <http://www.observatoriodemocratico.org/wp-content/uploads/2011/07/JURISPRUDENCIA-DE-LADEMOCRACIA_def_30_12_2012.pdf> [consulta: 10 de junio de 2015].

4 Aguiar, Asdrúbal. El derecho a la democracia. Caracas, Editorial Jurídica Venezolana/Observatorio IberoAmericano de la Democracia, 2008.

5 Diez de Velasco, Manuel. Instituciones de Derecho Internacional Público. 17a edición. Madrid, Editorial Tecnos, 2009. 
tales como la misma CDI o la Resolución 1080 de la Asamblea General de la OEA, no es totalmente descabellado argumentar que puede encontrarse en la convencionalidad interamericana los fundamentos teóricos y políticos de una obligación colectiva en la construcción de la forma y esencia democrática de los países de América. La institución de la corresponsabilidad, a pesar de tener base en distintos instrumentos de carácter vinculante, registra falencias fundamentales en cuanto a su modo de aplicación. Es a este respecto donde consideramos a la doctrina de la Responsabilidad de Proteger, o RtoP según su formulación inglesa ${ }^{6}$, como un marco de referencia apropiado para la comprensión y aplicación de una serie de mecanismos que doten de entidad y modo a la obligación colectiva democrática aquí defendida.

Esta doctrina tiene su génesis en el año 2001 por obra de la International Convention on Intervention and State Sovereignty, que se dio cita en Canadá y surgió como respuesta a lo señalado por el entonces Secretario General de las Naciones Unidas Kofi Annan en relación a varios crímenes cometidos en el siglo XX, al presentar el Reporte del Milenio del año 2000 a la comunidad internacional con ocasión de los recientes eventos de Yugoslavia y Ruanda. En éste, el diplomático preguntaba: "Si la intervención humanitaria es entonces un asalto inaceptable a la soberanía, ¿cómo debemos responder a Ruanda, a Srebrenica, a flagrantes y sistemáticas violaciones de derechos humanos que ofenden cada precepto de nuestra humanidad común?”7.

Si bien la doctrina de la Responsabilidad de Proteger apunta a hechos internacionalmente ilícitos ${ }^{8}$ asociados a los delitos llamados de megacriminalidad contenidos en el Estatuto de Roma y otros conexos y no exactamente a contemplaciones referentes a forma y sistema de gobierno - democrático en este caso-, postularemos que la forma democrática de gobierno es acaso la más sólida garantía que puede tenerse en el contexto americano para evitar la ocurrencia de situaciones tan atroces. De esta manera, construir la democracia es evitar activamente la ocurrencia de delitos de megacriminalidad por parte de los Estados americanos, y en tal sentido entendemos que la doctrina de la Responsabilidad de Proteger ofrece el marco adecuado para instrumentar la obligación colectiva de los gobiernos del hemisferio en la construcción de una forma de gobierno democrático, que prevenga la degeneración del tejido social y político de nuestros países a tal punto que se configuren delitos como el genocidio o crímenes de lesa humanidad.

Referido a Responsibility to Protect y también abreviada R2P.

Annan, Kof. Reporte del Milenio: Nosotros los pueblos. El rol de las Naciones Unidas en el siglo 21. Nueva York, Naciones Unidas, 2000. [en línea] <http://www.un.org/millennium/sg/report/full.htm> [consulta: 10 de junio de 2015].

8 Si se tiene como válida la premisa de que existe una obligación común a la sociedad internacional en su conjunto en evitar la ocurrencia de estos actos, la prevención de los mismos caería entonces dentro de la categoría marco de hecho internacionalmente ilícito. Sin embargo, esta discusión pertenece strictu sensu a la doctrina de la responsabilidad de proteger más que al tema que nos ocupa en esta instancia. 
En este mismo aspecto, y como consideración preliminar a los postulados que aquí realizaremos, cabrá recordar que en la Declaración Final de la Cumbre Mundial de Naciones Unidas del año 2005, la cual fue aprobada unánimemente por todos los Jefes de Estado y Gobierno allí presentes -incluidos sin excepción alguna los Estados Miembros de la OEA-, se afirmaba como necesaria la aplicación de la doctrina de la Responsabilidad de Proteger en sus párrafos 138 y 139. A este respecto, el Informe de 2009 del actual Secretario General de la Organización de Naciones Unidas, denominado Implementando la Responsabilidad de Proteger, dispuso que

Los Jefes de Estado y de Gobierno afirmaron unánimemente en la Cumbre que "cada Estado tiene individualmente la responsabilidad de proteger a su población del genocidio, los crímenes de guerra, la limpieza étnica y los crímenes de lesa humanidad". Acordaron también, que la comunidad internacional debería asistir a los Estados en el ejercicio de esa responsabilidad y en la construcción de sus capacidades de protección?

El punto central de dicho informe y de la doctrina de la Responsabilidad de Proteger, es que pertenece al Estado la obligación primaria de proteger a sus ciudadanos y que sólo cuando éste no pueda o no quiera honrar tal responsabilidad, se activará el deber subsidiario -aunque no por esto de menor entidad- de la sociedad internacional de ejercer putativamente la protección de los ciudadanos de otro Estado. Esto sin excluir la cooperación internacional en materia de construcción de capacidades de protección, que constituye uno de los pilares fundamentales de la doctrina, enfocada en la prevención.

Lo que aquí se pretende es trasladar los términos de referencia propios de la doctrina de la Responsabilidad de Proteger, integrando como forma de garantía más elevada y propia del contexto americano la protección y construcción de la democracia y el ejercicio de mecanismos colectivos para asegurar un pronto restablecimiento en caso de rompimiento del hilo constitucional. De esta manera, entenderemos que la responsabilidad primaria en la construcción y salvaguarda de la democracia es del Estado, pero si éste no puede o no quiere honrar tal obligación, la comunidad interamericana tiene el deber político, moral y jurídico de involucrarse activamente. De igual forma y en consistencia con lo que en adelante denominaremos la responsabilidad democrática compartida, los Estados Miembros de la OEA tienen el deber fundamental de cooperar en la construcción de capacidades democráticas.

9 KI-Moon, Ban. Implementing the Responsibility to Protect. Reporte del Secretario General. Documento A/63/677 del 12 de enero de 2009. [en línea] <http://es.unrol.org/files/SG_reportA_63_677_en.pdf> [consulta: 10 de junio de 2015]. 
A tal efecto, nos pasearemos en estas páginas por conceptos tales como la democracia tal como está diseńada en la CDI, el derecho a la democracia en el contexto americano y el debate que lo rodea como derecho fundamental o inclusive humano y cómo tales formulaciones políticas y jurídicas insertan el compromiso y la responsabilidad democrática compartida en el marco de las denominadas responsabilidades especiales ${ }^{10}$, que constituyen regímenes políticamente exigibles con fundamento jurídico en un determinado contexto social. De igual manera, ahondaremos sobre los fundamentos jurídicos que sustentan la 'americanización' de la Responsabilidad de Proteger y habilitan el marco de lo que aquí postularemos como responsabilidad democrática compartida, haciendo alguna precisión sobre su aplicabilidad y finalmente, cuál sería la situación de dicho planteamiento al confrontarlo con el Principio de No Intervención, el cual tiene amplia tradición en el contexto americano.

\section{La democracia prescriptiva en la Carta Democrática Intera- mericana}

Sartori entiende que "un sistema democrático está sustentado en una deontología democrática, y lo que la democracia es no puede separarse de lo que la democracia debería ser" ${ }^{\prime 1}$. De esta forma distingue el autor italiano entre lo que denomina la democracia prescriptiva, referido al deber ser de la democracia, y la democracia descriptiva, que atiende a su realidad empírica derivada de la práctica. En función de esto, podemos constatar que el régimen democrático diseñado por la CDI es, a todas luces, una enunciación prescriptiva, casi un desideratum. Tal cúmulo de características se encuentra presente a lo largo del texto del instrumento y es presentado como un elemento esencial de la democracia participativa. Destaca especialmente el artículo 2, que adelanta el carácter esencial de la democracia representativa, pareciendo indicar que -al menos en el contexto americano- sólo en democracia puede haber Estado de Derecho, signando además como apellido de esa democracia la cualidad de participativa ${ }^{12}$. De manera similar, otros artículos precisan los elementos fundamentales de la democracia participativa americana, tales como el artículo 3 (respeto a los DDHH, libre acceso y ejercicio del poder, celebración de elecciones secretas, periódicas, libres y universales, libertad de partidos políticos y separación e independencia de los poderes públicos), el artículo 4 (transparencia, probidad y responsabilidad en la gestión pública, respeto a los derechos sociales

10 Sobre este particular, ver Bukovansky, M., Clark, I., Eckersley, R. et al. Special Responsibilities: Global Power and American Power. Cambridge, Cambridge University Press, 2012.

1 SARTORI, Giovani. ¿Qué es la Democracia? 2a edición. Madrid, Taurus Ediciones, 2007.

12 Carta Democrática Interamericana. OEA/AG/RES.1838 (XXXI-O/O1) (2001), artículo 3. 
y a las libertades de comunicación y la sujeción gubernamental al estado de derecho) ${ }^{13} \mathrm{y}$ de igual forma los artículos 5 y $6^{14}$ que desarrollan en mayor detalle el artículo 3.

Así, rescatando lo dispuesto por el Juez García Ramírez en su voto separado en el caso Escher y otros vs. Brasil del año 2009, podemos identificar los componentes que dan la cualidad de democrático a un Estado en estricta sujeción a la Carta, que son entre otros:

La sujeción a la ley mediante la independencia de los poderes y la fiscalización de los actos del gobierno por órganos jurisdiccionales del Estado; surgimiento de los gobiernos mediante elecciones libres; incompatibilidad con el ejercicio de la democracia de la perpetuación en el poder o el ejercicio de éste sin plazo determinado o con manifiesto propósito de perpetuación; deber de los gobiernos de sostener un régimen de libertad individual y justicia social fundado en el respeto a los derechos humanos; protección judicial eficaz de los derechos humanos; contrariedad con el orden democrático de la proscripción política y sistemática; ejercicio de la libertad de prensa, información y expresión en tanto que condición esencial para la existencia del mismo sistema democrático; en fin, desarrollo de estructuras económicas que aseguren condiciones justas y humanas de vida para los pueblos ${ }^{15}$.

De esta forma, se dota de contenido a la expresión democracia participativa en el contexto de nuestro hemisferio. En tanto un determinado gobierno cumpla con todas estas características sin excepción alguna, estaremos en presencia de un régimen perfectamente democrático. No puede dejar de observarse que esta es una formulación prescriptiva que naturalmente está sujeta a carencias. Dichos déficits afectan principalmente a los habitantes de las Américas, quienes son a su vez los principales beneficiarios de lo dispuesto en la Carta en función del denominado Derecho a la Democracia. Éste los convierte en titulares de una serie de garantías asociadas al goce efectivo de todas las características incluidas en la CDI. Tales características son entonces entendidas como derechos-garantías que el ciudadano puede y debe exigir a su Estado en ejecución de los derechos que le reconocen los gobiernos signatarios por instrumento de la Carta Democrática Interamericana.

Carta Democrática Interamericana..., artículo 4.

Carta Democrática Interamericana..., artículos 5 y 6.

Corte Interamericana De Derechos Humanos. Caso Escher y otros vs. Brasil. 6 de julio de 2009. 


\section{La tesis de las responsabilidades especiales}

Con el ostensible avance de la conciencia colectiva en términos de gobernabilidad y estándares de vida que las sociedades americanas exigen de sus gobiernos, se ha creado en los ciudadanos de nuestro hemisferio la cultura de exigir la democracia a sus gobiernos y además la, hasta ahora tímida, práctica de monitorearse mutuamente entre los Estados. Es especialmente valioso encuadrar la situación de la responsabilidad democrática compartida americana en el contexto de la tesis de las responsabilidades especiales estipulada por Bukanovsky, Clark, Eckersley, Price, Reus-Smit y Wheeler, la cual estipula que es una modalidad de política que se sitúa en un punto de encuentro entre la igualdad soberana formal de los Estados, propia del orden mundial posterior a la Segunda Guerra Mundial, y, por otro lado, la concepción realista de las relaciones internacionales que versa sobre el libre juego de la política de poder y la existencia de recursos para aquellos que tienen poder en detrimento de aquellos que no lo tienen ${ }^{16}$. En este sentido creemos que las responsabilidades especiales, tales como las asociadas a la construcción de la infraestructura democrática en América, intentan mediar entre estos extremos. Como consecuencia de este compromiso, la responsabilidad política y jurídica se concreta en el marco de lo que la doctrina de las relaciones internacionales ha dado en llamar regímenes internacionales, de los cuales se ha dispuesto que:

Pueden ser entendidos como el resultado del comportamiento racional de los actores -principalmente estatales- que los crean. Los regímenes son necesarios en parte porque facilitan el logro de acuerdos, proveyendo información y reduciendo los costos de transacción en la política mundial. La interdependencia creciente entre los asuntos - mayor 'densidad de los problemas'- llevará a una mayor exigencia de regímenes. En tanto dichos espacios tengan éxito en proveer información [recíproca] de alta calidad, a través de procesos tales como la construcción de normas generalmente aceptadas o el desarrollo de relaciones entre los gobiernos, crearán la demanda de su propia continuación ${ }^{17}$.

Es particularmente importante rescatar este concepto de Keohane, por cuanto los regímenes internacionales, según desarrolla la dogmática de la interdependencia compleja en general, se consolidan alrededor de la maximización en la ganancia de beneficios colectivos $^{18}$. En la convergencia de tales intereses en un grupo de actores relativamen-

6 Bukovansky, Clark, Eckersley, et al, Special Responsibilities... op.cit.

17 Keohane, Robert. "The demand for international regimes", en International Organization, 1982, Vol. 36 (2), pp. 325-355. [en línea] <http://journals.cambridge.org/action/displayAbstract?fromPage=online\&a $\mathrm{id}=4281400>$ [consulta: 10 de junio de 2015].

18 En este contexto, la idea de 'beneficios' debe entenderse en el cariz que le otorga la escuela neoliberal de las 
te homogéneo en sus valoraciones sobre su interés nacional, el factor aglutinante por excelencia -además del incremento de los beneficios colectivos, naturalmente- son los valores que son comunes y a la vez son potenciados por la cooperación en tanto ésta se sostiene en el tiempo.

Si deseáramos argumentar la existencia de un régimen internacional en el hemisferio americano, cuyo valor central y elemento de ganancias a ser maximizado sea precisamente la democracia, no habría más que revisar el texto y espíritu de la Carta Democrática Interamericana. La importancia de destacar el carácter de régimen internacional del sistema democrático interamericano deviene precisamente de lo que aquí hemos apuntado con referencia a la tesis de las responsabilidades especiales. Estas son sin lugar a dudas propias del conjunto de Estados que han suscrito formalmente la democracia como única forma de gobierno en la cual se puede garantizar el pleno desarrollo del ser humano en todas sus potencialidades. Por esta razón y tratándose de un régimen especialísimo en el que han convenido estos Estados, lo que inicialmente parecía ser un compromiso unilateral de cada Estado en la promoción y defensa de la democracia cobra entonces un sentido colectivo. Ello debe traducirse en una vigilancia activa para mantener los estándares democráticos. Opinamos que tal obligación debe materializarse mediante la cooperación y la asistencia en la construcción de capacidades democráticas que habiliten una infraestructura socio-política donde la democracia pase del dicho al hecho, asumiendo la prevención como la mejor política para evitar la erosión democrática.

\section{La corresponsabilidad política en el contexto democrático}

Las bases legales y éticas de la responsabilidad especial de los Estados americanos con la democracia, entendida ahora como colectiva, pueden ser encontradas en distintos instrumentos tanto del sistema internacional como del americano, lo que a su vez le otorga exigibilidad por méritos propios. La misma Carta Democrática en sus considerandos hace clara referencia al carácter colectivo de los compromisos que se asumen por intermedio de ésta. Para muestra, lo siguiente:

CONSIDERANDO que la solidaridad y la cooperación de los Estados americanos requieren la organización política de los mismos sobre la base del ejercicio efectivo de la democracia representativa y que el crecimiento económico y el

relaciones internacionales. Ésta toma en cuenta a los grupos sociales y de poder que se encuentran insertos formal o informalmente en la estructura estatal o en el conglomerado social. Esto viene a apuntalar la idea de que son los grupos domésticos los que exigen más y mejor democracia en un bottom-up approach. 
Revista Tribuna Internacional

Volumen $4 \cdot \mathrm{N}^{0} 7 \cdot 2015 \cdot$ pp. 69-86

ISSN 0719-210X (versión impresa)/ISSN 0719-482X (versión en línea)

desarrollo social basados en la justicia y la equidad y la democracia son interdependientes y se refuerzan mutuamente ${ }^{19}$.

Destaca en esta sección el reconocimiento de la necesidad de la solidaridad y la cooperación para la construcción de la democracia representativa. Si consideramos lo anterior como cierto, esta cláusula parecería indicar que la democracia sólo puede ser construida colectivamente. De esta forma la misma Carta Democrática da carácter de necesidad -o podríamos argumentar que incluso lo preceptúa como obligación- al concurso de todos los Estados americanos para lograr el cometido común que se ha propuesto con ocasión de este régimen.

Podemos argumentar entonces que la responsabilidad en la construcción de la democracia en el hemisferio americano tiene carácter común y además exigible, en lo político y en lo jurídico. Para sustentar esto basta con revisar los instrumentos pertinentes al tema democrático en el contexto de la OEA, como lo son por ejemplo la Carta de la Organización de Estados Americanos de 1948, el Compromiso de Santiago de 1991, la Declaración de Nassau de 1992, la Declaración de Cartagena de Indias de 2009 o la misma Carta Democrática Interamericana de 2001, la cual dispone en sus considerandos sobre el carácter mancomunado de la responsabilidad democrática compartida y su implementación. En la Declaración de Nassau se acordó desarrollar mecanismos para proporcionar la asistencia que los Estados Miembros soliciten para promover, preservar y fortalecer la democracia representativa, a fin de complementar y ejecutar lo previsto en la resolución AG/RES. 1080 (XXI-O/91) ${ }^{20}$.

Sobre la cuestión de los mecanismos de implementación, exigibilidad y efectividad de la corresponsabilidad política y jurídica en la construcción de la democracia en las Américas, la Resolución AG/RES. 1080 (XXI-O/91) de la OEA puso el énfasis en la necesidad de que tal mandato compartido adquiriese efectividad entre los Estados signatarios. Esto puede ser evidenciado en el siguiente apartado de la misma resolución, el cual indica:

Que teniendo en cuenta la existencia generalizada de gobiernos democráticos en el hemisferio, es necesario darle efectiva aplicación al principio consignado en la Carta de que la solidaridad de los Estados americanos y los altos fines que con ella se persiguen requieren la organización política de los mismos sobre la base del ejercicio efectivo de la democracia representativa ${ }^{21}$.

\footnotetext{
Carta Democrática Interamericana..., considerando $6^{\circ}$.

Organización de Estados Americanos, Declaración de Nassau de 19 de mayo de 1992, AG/DEC. 1 (XXII-O/92) (1992).

21 Organización de Estados Americanos, Resolución AG/RES. 1080 (XXI-O/91), de 5 de junio de 1991.
} 
Consta ahora plenamente que la obligación colectiva y la responsabilidad compartida en la construcción del derecho a la democracia existe y debe ser además, por mandato del mismo sistema regional americano, exigible y efectiva. Sin embargo, brilla por su ausencia el mecanismo que le dé entidad a tal obligación. No existe hasta el momento una doctrina o práctica que adelante el cometido de honrar la responsabilidad especial de la capacitación democrática.

Fuera de los discursos fraternos que pretenden acercar ideológicamente a determinados gobiernos en el marco del sistema interamericano so pretexto de 'profundizar la democracia' u otros valores que le son artificialmente adjuntados, poco o ningún avance hemos observado en los años que han seguido a la adopción de la CDI por materializar la cooperación y asistencia recíproca en la instauración, mantenimiento y protección de la democracia. Es por esta razón que deseamos traer aquí a colación y ofrecer como alternativa una variación de la doctrina -relativamente reciente- de la Responsabilidad de Proteger, originada en Canadá a finales del año 2001 como respuesta al emplazamiento realizado por Kofi Annan con respecto a las atrocidades de Srebrenica y Ruanda en la década de 1990-2000.

\section{La americanización de la Responsabilidad de Proteger}

La misión del Estado moderno consiste en la protección, salvaguarda y provisión del mejor nivel de vida posible a sus ciudadanos. Siguiendo el espíritu de la Carta Democrática, podemos argumentar que esto sólo es posible en la medida en que se verifiquen los parámetros de democracia formal que dicho instrumento consagra. De igual manera, cabe esperar de los Estados americanos que subordinen nociones como la soberanía al respeto de los DDHH. En este contexto, las respuestas a problemas modernos, como la implementación de la democracia en el hemisferio americano, deben ser forzosamente innovadoras y romper con los parámetros tradicionales de acción de la sociedad internacional tal como la conocemos hasta ahora. Aquellos mecanismos que eleven el nivel de compromiso de los Estados en el marco de la protección de los DDHH deben ser considerados no desde la actitud restrictiva y absoluta del concepto de la soberanía clásica o westphaliana, sino desde una revisión crítica de la labor y misión del Estado. En este orden de ideas, el reporte de la Comisión Internacional sobre Intervención y Soberanía Estatal, de la cual se originó la Responsabilidad de Proteger, precisó que:

El debate actual sobre las intervenciones con propósitos de protección humana también tiene lugar en un contexto histórico, político y legal de evolución de los estándares de conducta para los Estados y los individuos, incluyendo el desarrollo de normas nuevas y más firmes y mecanismos para la protección de 
los derechos humanos. Los derechos humanos se han convertido ahora en una parte central del Derecho Internacional, y el respeto por los DDHH ha pasado a ser un tema central y una responsabilidad en las relaciones internacionales ${ }^{22}{ }^{23}$

En el caso específico del contexto americano y de la democracia como responsabilidad compartida, aparece como un mandato asumir un "espíritu de renovación signado por la idea de la perfectibilidad”, en palabras de Aguilar $^{24}$, frente al deterioro y debilitamiento experimentado por la democracia. Este mismo autor destaca, acerca de la existencia de un orden público democrático, según manifestó la Corte Interamericana de Derechos Humanos, en el caso Gelman, "se reitera en la exigencia del empleo necesario de medios adecuados para enfrentar amenazas o actos de violencia con instrumentos y procedimientos legítimos y dentro del orden jurídico propio de una democracia" ${ }^{25}$. Así, para llenar el vacío existente en la convencionalidad americana sobre la existencia de un recurso efectivo para asegurar el cumplimiento de la obligación de los Estados en la construcción colectiva de la democracia, ofrecemos la tesis de la Responsabilidad de Proteger como orientación.

Esta doctrina, en sus principios básicos, estima que "la soberanía estatal implica responsabilidad, y que la responsabilidad primaria por la protección de los pueblos resta en el Estado mismo"26. De igual manera, el Secretario General de las Naciones Unidas, Ban Ki-Moon, apuntó, en su reporte del año 2009 llamado Implementing the Responsibility to Protect, que la comprensión de que la responsabilidad primaria en la protección de los DDHH compete al Estado constituía el primer y más fundamental pilar de esta norma. Esto es conteste con lo observado sobre la naturaleza dual del compromiso democrático que es inicialmente individual de cada Estado, pero que también tiene una dimensión colectiva, producto de la responsabilidad especial que se forma en el marco del régimen internacional para la promoción y protección de la democracia en el hemisferio americano.

22 Evans, Gareth y Sahnoun, Mohamed. The Responsibility To Protect. Ottawa, International Development Research Centre, 2001. [en línea] <http://responsibilitytoprotect.org/ICISS\%20Report.pdf> [consulta: 10 de junio de 2015].

23 Será cabal aclarar con respecto a esto, que la noción de intervención ha de ser entendida en su acepción más lata. Si se cae en la ligereza de entender por intervención sólo la violación flagrante de la soberanía, el territorio y la independencia política de un Estado, entonces entraremos en el círculo vicioso de la política que caracterizó el orden mundial existente entre 1945 y el fin de la Guerra Fría, lo que sería un despropósito al cometido del presente trabajo. De esta forma, la intervención como institución deberá ser entendida como integrada por la asistencia, la colaboración y la cooperación internacional a efectos de la consecución de un determinado logro, sin excluir en los casos de atrocidades masivas que violen los derechos humanos la adopción de medidas colectivas de mayor entidad.

24 Aguiar, La Democracia en la jurisprudencia..., op.cit.

25 Aguiar, La Democracia en la jurisprudencia..., op.cit.

26 Evans y Sahnoun, The Responsibility..., op.cit. 
La institución de la R2P está fundamentalmente destinada a evitar o detener la comisión o complicidad por parte del Estado de graves crímenes masivos contra los derechos humanos. En este sentido pone como presupuestos para su aplicación que ocurran o puedan materializarse por la acción u omisión estatal ante delitos como el genocidio, la limpieza étnica, los crímenes de guerra o crímenes de lesa humanidad. Esto queda plenamente explicado en su segundo principio básico, el cual dispone que:

Donde una población sufra serios daños, como resultado de guerra civil, insurgencia, represión o falla del Estado, y el Estado en cuestión no quiera o no pueda detener o evitar la situación, el principio de la no intervención cede al de la responsabilidad internacional de proteger ${ }^{27}$.

Si bien el presente trabajo no busca discurrir sobre este tipo de supuestos al versar sobre construcción de capacidades democráticas, y no sobre violaciones masivas a los derechos humanos, sí hemos planteado que la perversión de la democracia o la omisión de implementarla con arreglo a los parámetros formales regulados por la Carta Democrática conduce eventualmente a la erosión del tejido y el orden social. Esta precondición es el "caldo de cultivo" idóneo para situaciones como las planteadas en el informe de la ICISS, referentes a atrocidades masivas que violen de forma grosera e inadmisible los derechos humanos. Así, sostenemos que la democracia es efectivamente la mejor garantía que puede haber contra la ocurrencia de situaciones como guerras internas o insurgencias o la degeneración de la función del Estado, convirtiéndolo en verdugo en vez de defensor de los DDHH.

La misma Carta Democrática Interamericana hace eco de tal visión cuando resalta en su primer considerando que "la democracia representativa es indispensable para la estabilidad, la paz y el desarrollo de la región". De esta manera, si compaginamos esta noción de la democracia representativa como forma de garantía más elevada contra la violación de los $\mathrm{DDHH}$, en el entendido que la forma democrática es un derecho de los pueblos de las Américas, según la tesis adoptada unánimemente por los Estados Miembros de Naciones Unidas -los Estados americanos entre ellos- en el Documento Final de la Cumbre Mundial de 2005 en sus párrafos 138 y 139, referentes al deber de la "comunidad internacional de asistir a los Estados en el ejercicio de esa capacidad y en la construcción de capacidades de protección" 28 , nos encontramos con que puede integrarse el elemento democrático, en función de la responsabilidad especial de los Estados de nuestro hemisferio en ese respecto, con las posturas de la R2P. Tanto porque no se registra incompatibilidad alguna entre la formulación de esta institución y el compromiso con el derecho a la democracia, como por la aceptación explícita que hicieran de

27 Evans y Sahnoun, The Responsibility..., op.cit. Una versión más limitada de ese catálogo de supuestos se aprobaría con posterioridad en el Documento Final de la Cumbre Mundial del año 2005, párrafos 138-140.

28 Naciones Unidas, AG/RES/60/1. Documento Final de la Cumbre Mundial de 2005. 
Revista Tribuna Internacional

Volumen $4 \cdot \mathrm{N}^{0} 7 \cdot 2015 \cdot$ pp. 69-86

ISSN 0719-210X (versión impresa)/ISSN 0719-482X (versión en línea)

la norma todos los Estados Miembros de la OEA en la Cumbre Mundial de Naciones Unidas del año 2005.

\section{Los tres pilares y la responsabilidad de prevenir}

A efectos de presentar una visión más completa de lo que constituye la R2P y de cómo puede ésta ser revisada e implementada con las variaciones pertinentes al marco de las Américas, conviene revisar el reporte del Secretario General de la ONU del año 2009 del que anteriormente habíamos hecho mención. Tal instrumento, en línea con lo dispuesto en 2001 con el reporte de la ICISS y la Declaración Final de la Cumbre Mundial de 2005, delinea tres pilares básicos de la Responsabilidad de Proteger, que versan sobre las responsabilidades de protección del Estado o primer pilar, la asistencia internacional y la construcción de capacidades, llamada en el texto el segundo pilar, y por último el tercer pilar, referente a la respuesta oportuna y decisiva cuando se presenten las situaciones que den pie a la activación de la Responsabilidad de Proteger.

El primer pilar versa sobre las ideas que expusiéramos referentes a la responsabilidad primaria del Estado en la protección de sus nacionales frente a violaciones masivas y atroces de los DDHH. En el marco de la propuesta que adelantamos, dicho lenguaje quedaría trocado por la responsabilidad primaria del Estado en la protección del derecho a la democracia. También, el primer pilar, según advierte Ki-Moon, está referido a la obligación concomitante de la sociedad internacional en la promoción y defensa de tal plataforma de protección, especialmente en los casos donde el Estado no pueda o no quiera cumplir con ese mandato ${ }^{29}$.

Sobre el segundo pilar -acaso el más importante en la temática que exploramos-, Evans y Sahnoun (2001) han dicho que "la responsabilidad de proteger implica la responsabilidad de prevenir". Sobre este particular, disponen que es momento de cerrar la brecha entre el apoyo retórico a la prevención y un compromiso tangible y verdadero. En el texto de su informe de 2009, el Secretario General de Naciones Unidas precisó que el segundo pilar aborda:

(b) el compromiso de la comunidad internacional de asistir a los Estados en el cumplimiento de esas obligaciones. Busca nutrirse de la cooperación de los Estados Miembros, de los acuerdos regionales y subregionales, de la sociedad civil y el sector privado, así como en las fortalezas institucionales y ventajas comparativas del sistema de Naciones Unidas. (...) el segundo pilar es crítico

29 KI-Moon, Implementing the Responsibility..., op.cit. 
para el forjamiento de políticas, procedimientos y prácticas que puedan ser consistentemente aplicadas y ampliamente apoyadas ${ }^{30}$.

Es especialmente revelador, a efectos de la proposición que aquí hacemos sobre la adopción de un marco de responsabilidad democrática común, el hecho de que el mismo Secretario General destaque el rol de los acuerdos regionales, la OEA entre ellos, en el mandato de construcción de capacidades para la defensa de los DDHH, o en el contexto especialísimo de régimen democrático americano, la construcción de capacidades democráticas y el respeto del derecho a la democracia. En consonancia con esto, podemos argumentar que si se demostrara la vocación política de apoyar los esfuerzos regionales para atender las causas primarias de los problemas y sus catalizadores, podría ganarse mayor credibilidad y basamento para la implementación de formulaciones como las que aquí hemos adelantado. Deberá notarse que uno de los principales problemas de la prevención, tal como lo hemos reinterpretado, es que algunos Estados son reacios a aceptar cualquier tipo de medidas preventivas sugeridas o puestas en marcha por la sociedad internacional, incluso aquellas de carácter menos injerencista. Podríamos argumentar que tal miedo obedece a que en su criterio cualquier internacionalización de los problemas podría resultar en una interferencia externa que eventualmente podría conducir a una intervención que amenace la integridad e independencia política de dicho Estado.

Por último, las medidas que se aplican cuando la prevención falla, de acuerdo a los documentos existentes en materia de R2P, son las que constituyen el tercer pilar de ésta. Las respuestas oportunas y decididas son entonces aquellas que, de acuerdo a los principios de progresividad, autoridad competente del Consejo de Seguridad de la ONU, último recurso y justa causa, se toman cuando las medidas de prevención han sido agotadas. Sin embargo, insiste el informe, en lo referente a las prioridades que deben tenerse cuando se considere esta opción, que "la prevención es la dimensión más importante de la responsabilidad de proteger y esta opción siempre deberá ser agotada antes de que se contemple la intervención" ${ }^{31}$. Esta visión está en profunda consonancia con lo que consideramos como el fundamento de la doctrina de prevención que pretende ser la responsabilidad democrática compartida en las Américas.

KI-Moon, Implementing the Responsibility..., op.cit.
Evans y SaHnoun, The Responsibility..., op.cit. 


\section{La responsabilidad democrática como régimen complemen- tario a la sección IV de la Carta Democrática Interamericana}

La sección IV de la Carta Democrática Interamericana dispone el conjunto de opciones con las que cuentan tanto el Estado como el resto de la comunidad americana respecto a la situación de peligro, deterioro o quebrantamiento de la institucionalidad democrática en los términos prescriptivos de la sección I. Así, se contempla la posibilidad de pedir asistencia al Secretario General de la OEA o a su Consejo Permanente (artículo 17), pedir los buenos oficios del Secretario General para la consideración colectiva de la situación (artículo 18) y como medida de mayor gravedad, como amonestación política, la suspensión del Estado en cuestión de su participación en la organización (artículo 19). Sin embargo, el artículo 20 en su párrafo tercero dispone que:

Si las gestiones diplomáticas resultaren infructuosas o si la urgencia del caso lo aconsejare, el Consejo Permanente convocará de inmediato un período extraordinario de sesiones de la Asamblea General para que ésta adopte las decisiones que estime apropiadas, incluyendo gestiones diplomáticas, conforme a la Carta de la Organización, el derecho internacional y las disposiciones de la presente Carta Democrática ${ }^{32}$.

Es visible que el artículo 20, aunque con un lenguaje muy tímido en atención al respeto al principio de igualdad soberana de sus signatarios, quiere significar que las medidas que se pueden aplicar cuando se verifique el rompimiento del hilo democrático no se agotan con las previstas en la carta. Prueba de esto lo encontramos en la mención que hace al derecho internacional como potencial catálogo de opciones a tomar cuando la democracia se vea en serio peligro o deteriorada de forma ostensible. $Y$ es precisamente en la ventana que se abre con ocasión de esta disposición, que sostenemos que la asimilación de la Responsabilidad de Proteger como institución emergente de derecho internacional, sirve como una de las medidas aplicables en aquellas situaciones donde un Estado signatario de la CDI vea comprometida la estabilidad y solidez de su proceso democrático. Obviamente, y tal como ya hemos seńalado, la doctrina de la R2P precisaría ajustes conceptuales para podérsela aplicar en el marco americano, sin embargo, tales ajustes no suponen un cambio radical a su contenido, sino sencillamente la adopción de la noción de que la democracia -en los términos formales y prescriptivos en que la consagra la misma Carta Democrática- constituye una garantía superior y efectiva para evitar violaciones masivas de derechos humanos.

32 Carta Democrática Interamericana..., artículo 20. 
Señalando, como hemos hecho, que la faceta más útil de la norma de la R2P es la prevención, conviene analizar dentro de la misma doctrina, cuáles son esas medidas enmarcadas en el segundo pilar que coadyuvarán en la construcción de capacidades. Tales medidas deben, por fuerza, ser en primera instancia adoptadas por el Estado, pero es fundamental que cuenten con el apoyo, cooperación y asistencia de los demás miembros de este régimen de responsabilidad especial democrática, acatando así lo dispuesto por el primer y segundo pilar de la R2P.

Las medidas aplicables que plantea la Responsabilidad de Proteger tal como la desarrolla el Secretario General de la ONU en su informe de 2009 y que aquí presentamos a título de régimen complementario de las regladas en la CDI, enmarcadas en el derecho internacional existente, serían entonces:

- El fomento de capacidades de análisis en el marco gubernamental e inter-gubernamental que consideren en su justa dimensión a las potencialidades de conflicto interno. Especialmente cuando éstas amenacen con atentar contra la forma y esencia democrática de la sociedad y su gobierno. Esta medida de cooperación podría verse satisfecha con la adopción de mecanismos de intercambio de información y de asesoramiento de riesgo, emprendida colectivamente para el adecuado manejo de conflictos internos que tiendan al incremento de tensiones que eventualmente podrían atentar contra la gobernabilidad y los estándares democráticos de un Estado.

- La cooperación en la construcción de capacidades de mediación local. Tal construcción supone la formación o fortalecimiento de instituciones y procesos, en el gobierno y la sociedad civil, que puedan cooperar en la solución de disputas, promoción de reconciliación o mediación en asuntos específicos.

- Instrumentación común de plataformas que permitan el consenso y el diálogo. Referida a la provisión de espacios neutrales o foros regionales para atender situaciones que amenacen el orden democrático. El informe del Secretario General destaca la labor de los Estados americanos en el establecimiento de espacios de 'diálogo democrático' como parte del proceso de gobernabilidad, lo cual indica que ésta y las demás medidas que aquí proponemos no son en lo absoluto incompatibles con el actual sistema democrático americano.

- Estímulo a la resolución regional de las controversias. Esto implica la construcción de lo que el reporte denomina infraestructura de paz. Así, aquellos temas con tendencia a generar tirantez socio-política en la ciudadanía - potenciales causas de erosión democrática- tales como disputas por la titularidad de tierras, disponibilidad de recursos, religión, etnicidad o sucesión en los liderazgos pueden y deben ser tratados, con el debido respeto a la soberanía y esfera doméstica de los Estados, en la agenda de los organismos regionales, o que en su defecto los miembros de tales 
Revista Tribuna Internacional

Volumen $4 \cdot \mathrm{N}^{0} 7 \cdot 2015 \cdot$ pp. 69-86

ISSN 0719-210X (versión impresa)/ISSN 0719-482X (versión en línea)

organismos cooperen con el Estado en cuestión para potenciar sus capacidades de gestión efectiva de los conflictos.

- Construir capacidad para replicar capacidad. Esta última serie de medidas, reinterpretadas desde el contexto de la acción colectiva en el hemisferio americano, supone que las capacidades antes descritas deben ser asimiladas por la sociedad para poder ser replicadas en venideras generaciones, quienes a su vez podrán prevenir o atender las situaciones de erosión que se presenten. Tal asimilación debe ocurrir por intermedio de cooperación estrecha entre los Estados y otras instituciones como universidades, sociedad civil e instituciones de servicio público.

La idea que sustenta en general a las perspectivas de la responsabilidad democrática como versión 'americanizada' de la Responsabilidad de Proteger es el diseńo de iniciativas, programas y mecanismos destinados específicamente a la construcción colectiva de infraestructura democrática y capacidades políticas y legales en el marco de las sociedades, para hacerlas menos propensas a tomar los senderos que conducen a situaciones como crisis de ingobernabilidad o rompimientos del orden y esencia democráticos.

Como precisión final, es menester precisar que estas medidas en ningún momento coluden con la independencia política, la integridad territorial ni con ninguno de los bienes jurídicos superiores que la institución de la soberanía protege. La intención de centrar el presente trabajo únicamente en el segundo pilar de la responsabilidad de proteger no es casual. Si se considera la situación actual del Principio de No Intervención en asuntos domésticos consagrado por la Carta de la OEA y se complementa con la declaración anexa a la resolución AG/RES/2521/XXV de la Asamblea General de las Naciones Unidas, es sencillo constatar que los criterios para determinar qué es una injerencia en los asuntos internos de un Estado son sumamente ambiguos, lo que tampoco es casual. Los Estados -y especialmente los de nuestro hemisferio- son particularmente celosos de su soberanía, que aún tiene en la mente de los gobernantes y decisores un cariz sumamente clásico.

Precisamente por esa concepción westphaliana de la soberanía que aún impera en nuestro continente es que postulamos la tesis de la responsabilidad democrática compartida, en términos de construcción de capacidades. La idea que subyace a esta renuncia a mecanismos más agresivos de protección de la democracia americana, es que la mejor manera de combatir la degeneración de la democracia o las falencias de la forma democrática en los gobiernos del continente americano es precisamente la prevención colectiva. 


\section{Conclusiones}

En función de las consideraciones expuestas al comienzo de este trabajo, podemos concluir que el compromiso pretendidamente individual de los gobiernos americanos con la democracia no es forzosamente tal. El planteamiento de la corresponsabilidad política, en el marco del régimen internacional conformado por el sistema democrático interamericano, nos da a entender que un Estado democrático rodeado de Estados que no lo sean, está condenado a fracasar y por esto es responsabilidad de cada gobierno la cooperación y asistencia en la construcción de capacidades democráticas que aseguren formas de gobierno sólidas y estables, respetuosas de los derechos humanos y especialmente del derecho a la democracia, que los Estados americanos han reconocido a sus habitantes. Esto por cuanto las realidades actuales de la globalización y el intercambio casi instantáneo de información por vía digital han elevado el desafío de la gobernabilidad para los decisores estatales, acercando elementos de comparación al ciudadano y otorgándole conciencia de su propia calidad de vida.

Para poder cumplir entonces con el derecho a la democracia y hacerlo colectivamente -que en nuestro criterio es la única manera sustentable de lograrlo-, debemos ampliar el rango de acción y poderes del régimen democrático interamericano, sea por vía de la OEA o de mecanismos especialmente diseñados al margen de tal organización. Las Américas tienen derecho a la democracia, y es entonces deber de sus gobiernos el materializar tal perspectiva y más aún, vigilarse recíprocamente en el cometido de ese logro, no para reprocharse o tomar medidas que atenten contra la soberanía de los Estados, sino para tenderse una mano de hermandad que asegure el gozo efectivo de la democracia para los habitantes del hemisferio.

Como mecanismo útil, apropiado y sobre todo de sencilla implementación en la consecución de esa meta, planteamos aquí una derivación de la norma de la responsabilidad de proteger. Esta es a nuestro juicio apropiada en atención a sus fines, lícita con miras a la legalidad interna y a la convencionalidad internacional, respetuosa en lo tocante a la independencia política y soberanía de los Estados y, sobre cualquier otra consideración, necesaria y largamente debida a los pueblos de las Américas, los cuales, en virtud de su tradición histórica y conciencia política, no deben ni pueden aceptar menos que la democracia que para ellos mismos han elegido, la cual consta en sus contenidos mínimos en la CDI. Sin embargo y como palabra de aliento, tal instrumento marca sólo el inicio del camino, ya que la meta es lejana y acaso imposible de alcanzar pero no por ello menos digna de ser perseguida. 